\title{
Liquid Scanning Transmission Electron Microscopy of Individual Quantum Dots Labeling Epidermal Growth Factor Receptors in Whole Cells
}

\author{
M. J. Dukes*, D.B. Peckys**,*** and N. de Jonge**,**** \\ *Vanderbilt University, Department of Chemistry, 7330 Stevenson Center, Station B 351822 \\ Nashville, TN 37235 \\ **Oak Ridge National Laboratory, Materials Science and technology Division, 1 Bethel Valley \\ Rd., Oak Ridge, TN 37831-6064 \\ ***University of Tennessee, Center for Environmental Biotechnology, 676 Dabney Hall \\ Knoxville, Tennessee 37996-1605 \\ $* * * *$ Vanderbilt University Medical Center, Department of Molecular Physiology and \\ Biophysics, 702 Light Hall, 2215 Garland Ave., Nashville, 37232-0615
}

Our understanding of molecular-scale cellular processes has increased as new methods for imaging cellular components have been developed [1]. Liquid scanning transmission electron microscopy (STEM) is a novel technique for imaging whole cells in liquid, with nanometer resolution on tagged proteins. Eukaryotic cells are grown on silicon microchips each containing an ultra-thin and electron-transparent silicon nitride ( $\mathrm{SiN})$ window. A microchip with attached cells is first imaged with light microscopy, see Fig. 1A. The microchip is then assembled into a micro-fluidic chamber together with a second microchip containing a spacer, and placed in the vacuum of the electron microscope. The cells are imaged with the scanning beam of the STEM (Fig. 1B). Liquid STEM is capable of imaging nanoparticles within a water layer of several micrometers thickness by exploiting the enhanced contrast of high-atomic number nanoparticles against low-atomic number materials [2]. Unlike transmission electron microscopy (TEM) of biological samples (e.g., TEM of stained and embedded thin sections, or cryo-EM), liquid STEM is capable of imaging intact cells, while maintaining nanometer resolution. Samples are prepared analogously to light microscopy samples. Cells are labeled live and then fixed with glutaraldehyde, after which no further sample fixation or staining is performed. Artifacts typically seen in EM samples due to dehydration, post-staining, freezing, or sectioning are thus avoided [3].

In this study, the ability to image quantum dots (QDs) in whole cells using liquid-STEM was investigated. COS7 fibroblast cells were cultured on silicon microchips containing a 50 x 200 $\mu \mathrm{m}$ area SiN window (Protochips Inc, NC). The epidermal growth factor (EGF) receptors were labeled using EGF conjugated to $655 \mathrm{~nm}$ emitting QDs (EGF-QD 655 ). After a five-minute incubation with EGF-QD 655 , the cells were washed, fixed and imaged with wide-field light microscopy directly on the microchip (Fig. 2A and 2B). Next, the microchip was enclosed in the sample holder and STEM images were recorded. In the liquid STEM image of Fig. 2C, individual QDs are visible as yellow-green spots distributed throughout the cellular membrane. Cellular contours can be seen as regions of blue shading. A resolution of $3 \pm 1 \mathrm{~nm}$ was determined from line scans over of six QDs. This value is comparable to the resolution obtained on gold nanoparticles in a similar sample [2].

These results demonstrate that liquid STEM is capable of imaging biologically targeted QDs on whole cells in a liquid environment, with nanometer resolution. The STEM images were correlated with fluorescence images of the same region, establishing that liquid STEM can be utilized for correlative imaging studies. This opens the possibility of multiplexing studies 
utilizing different sizes of QDs to label multiple cellular targets in a native (liquid) state environment.

\section{References:}

[1] A. Leis et al., Trends Biochem. Sci., 34 (2009) 60

[2] N. de Jonge et al., Proc. Natl. Acad. of Sci.106 (2009) 2159

[3] D.B. Peckys, et al., PLoS One 4 (2009) e8214.

[4] We are grateful to D.W. Piston, G.J. Kremers, S. Head, and Protochips Inc.. A Portion of this research was conducted at the SHaRE User Facility, which is sponsored by the Division of Scientific User Facilities, Office of Basic Energy Sciences, U.S. Department of Energy. Research supported by Vanderbilt University Medical Center, and NIH grant R01GM081801.
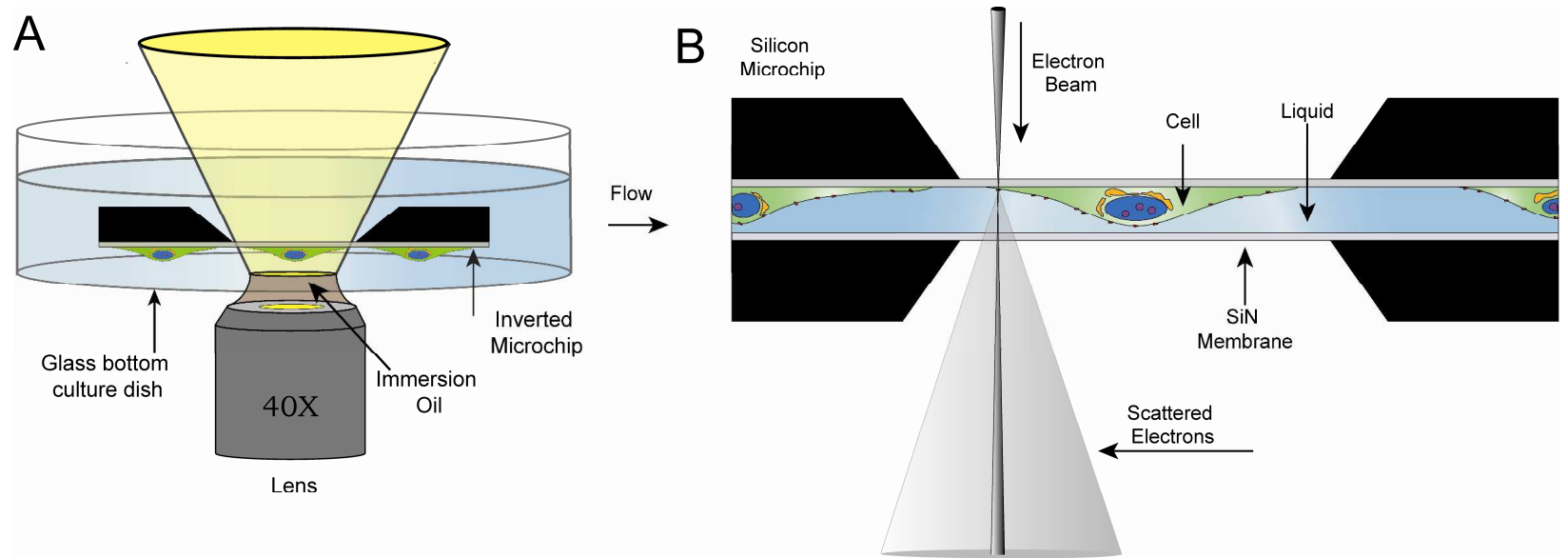

Figure 1: Correlative light microscopy and liquid STEM. (A) Schematic of setup for light microscopy imaging. A microchip containing cells is placed up-side-down in a glass bottom culture dish and imaged using an oil-immersion lens. (B) Schematic of setup for liquid STEM. A second microchip is used to form the bottom of a micro-fluidic chamber. The microchips are separated by microsphere spacers (not shown). The chamber is placed in a custom sample holder
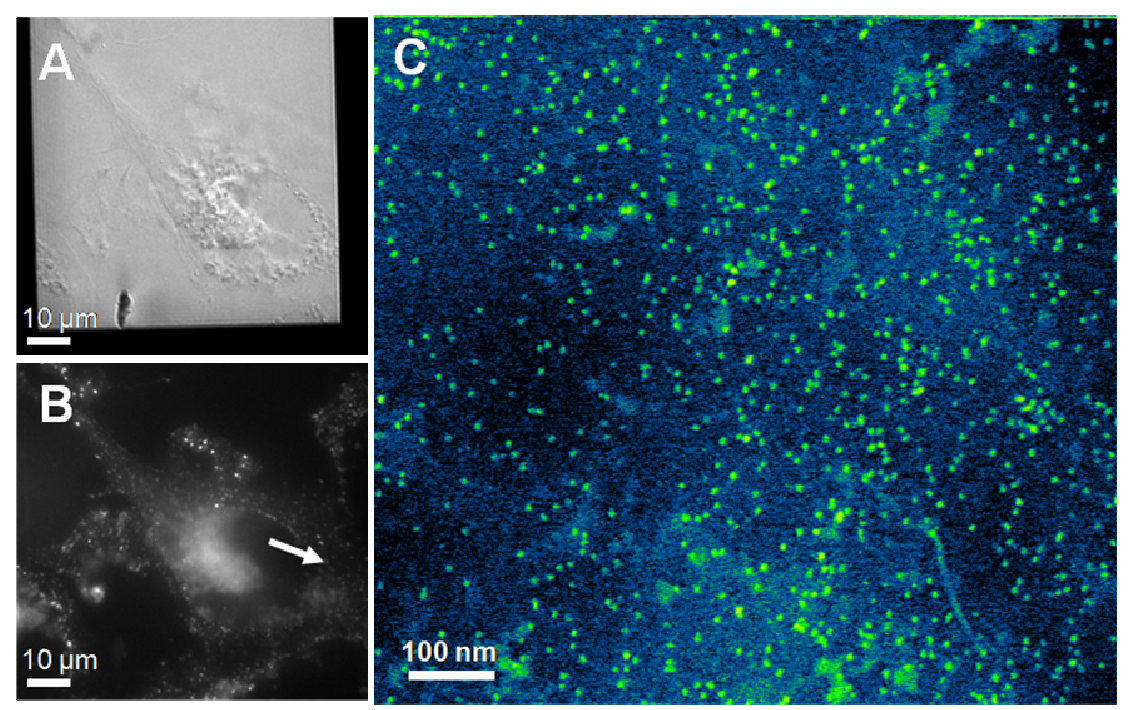
allowing the flow of liquid to and from the specimen. Contrast is obtained on nanoparticle protein labels.

Figure 2: Light micrscopy and liquid STEM of a COS7 cell with EGF receptors labeled with QDs. (A) Direct interference contrast image of labeled cell (B) Fluorescence image of labeled cell. The arrow indicates the region imaged with liquid-STEM (C) Liquid STEM image. The QDs are visible as bright yellow-green oval shapes distributed throughout the cell. Cellular contours appear as regions of blue shading. 